\title{
The Hippocampus and Dorsolateral Striatum Integrate Distinct Types of Memories through Time and Space, Respectively
}

\author{
- $J$ Janina Ferbinteanu \\ Departments of Physiology and Pharmacology, and Neurology, SUNY Downstate Medical Center, Brooklyn, New York 11203
}

Several decades of research have established that different kinds of memories result from the activity of discrete neural networks. Studying how these networks process information in experiments that target specific types of mnemonic representations has provided deep insights into memory architecture and its neural underpinnings. However, in natural settings reality confronts organisms with problems that are not neatly compartmentalized. Thus, a critical problem in memory research that still needs to be addressed is how distinct types of memories are ultimately integrated. Here we demonstrate how two memory networks, the hippocampus and dorsolateral striatum, may accomplish such a goal. The hippocampus supports memory for facts and events, collectively known as declarative memory and often studied as spatial memory in rodents. The dorsolateral striatum provides the basis for habits that are assessed in stimulus-response types of tasks. Expanding previous findings, the current work revealed that in male Long-Evans rats, the hippocampus and dorsolateral striatum use time and space in distinct and largely complementary ways to integrate spatial and habitual representations. Specifically, the hippocampus supported both types of memories when they were formed in temporal juxtaposition, even if the learning took place in different environments. In contrast, the lateral striatum supported both types of memories if they were formed in the same environment, even at temporally distinct points. These results reveal for the first time that by using fundamental aspects of experience in specific ways, the hippocampus and dorsolateral striatum can transcend their attributed roles in information storage.

Key words: hippocampus; striatum; cognitive memory; habits; functional principles of memory circuits; memory integration; memory systems

\section{Significance Statement}

The current paradigm in memory research postulates that different types of memories reflected in separate types of behavioral strategies result from activity in distinct neural circuits. However, recent data have shown that when rats concurrently acquired in the same environment of hippocampal-dependent spatial navigation and striatal-dependent approach of a visual cue, each of the two types of memories became dependent on both the hippocampus and dorsolateral striatum. The current work reveals that the hippocampus and dorsolateral striatum use distinct and complementary principles to integrate different types of memories in time and space: the hippocampus integrates memories formed in temporal proximity, while the lateral striatum integrates memories formed in the same space.

\section{Introduction}

The multiple memory systems theory postulates that different types of memories result from the activity of distinct brain

\footnotetext{
Received May 6, 2020; revised Sep. 4, 2020; accepted Sep. 18, 2020.

Author contributions: J.F. designed research; J.F. performed research; J.F. analyzed data; J.F. wrote the paper.

This work has been supported by National Institutes of Health Grant MH-115421. I thank Kasey Stern for work on this project during her 2019 summer internship in my laboratory, and Frank Barone for access to equipment in his laboratory. I also thank Drs. Robert McDonald, Bryan Devan, Mark Packard, Jarid Goodman, and Norman White for comments on previous versions of the manuscript.

The author declares no competing financial interests.

Correspondence should be addressed to Janina Ferbinteanu at janina.ferbinteanu@downstate.edu.

https://doi.org/10.1523/JNEUROSCI.1084-20.2020

Copyright $\odot 2020$ the authors
}

circuits with different properties and dynamics. The operational principle of the memory systems is considered to be independent parallelism, meaning that although information flows through all memory networks at the same time, processing within a given system occurs autonomously and each network supports one kind of memory (Squire et al., 1993; Squire, 2004; White et al., 2013). This principle was formulated after numerous experiments in both animals and humans had shown that damage to one circumscribed memory circuit caused deficits in one of two behavioral tasks, each testing a distinct type of memory, while damage to a different memory circuit resulted in the opposite pattern. Based on numerous experiments of this kind, it is widely accepted that declarative memory, which includes spatial represe- 
A Spatial navigation with serial reversals

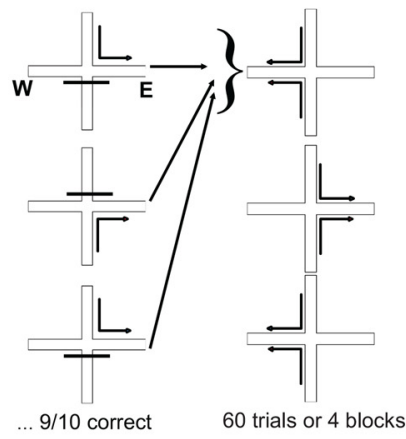

Cue Response
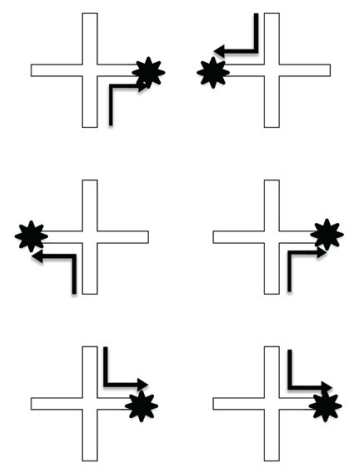

45 trials in random sequence

B
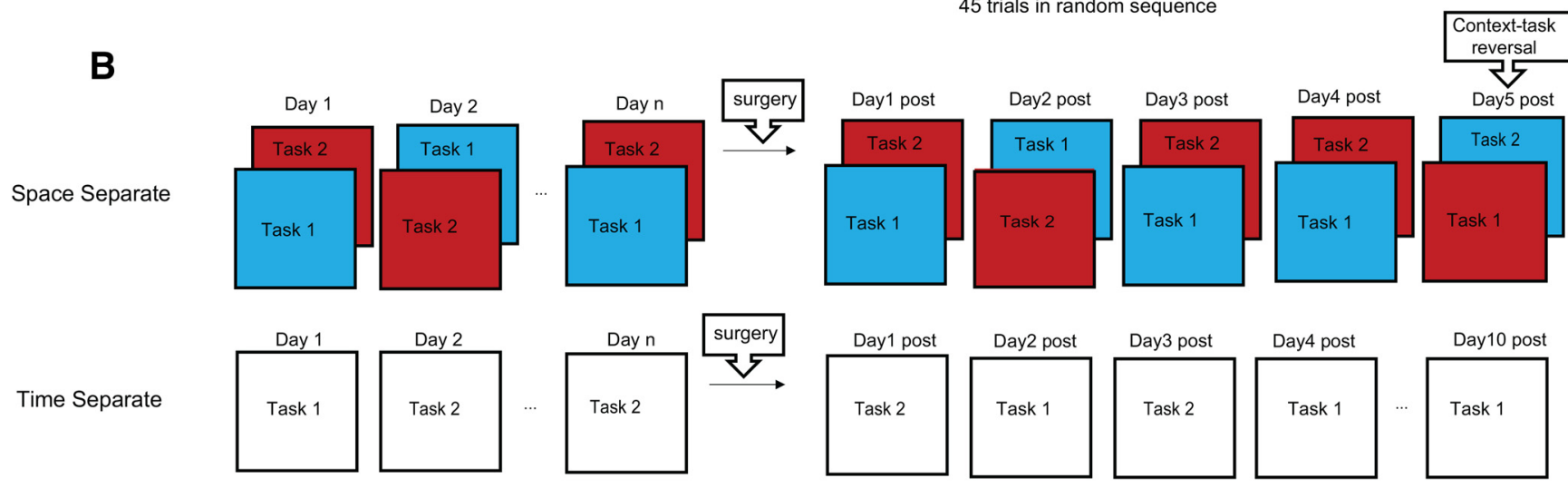

Figure 1. Behavioral paradigm and experimental design. $A$, The experiment used two distinct tasks: spatial navigation with serial reversals (left) and cue response (right). In both tasks, the animals started either in the North or South arm of a plus maze apparatus and had to find food in either the East or the West arms. On any given trial, the unused start arm was blocked. In the spatial navigation task, the food could be found based on its location, which was consistent until the rat chose the correct arm nine times correct in a sequence of 10 trials. At that point, the location of the food was switched to the other arm and a new block of trials started. Each session was constituted of four blocks to a total of maximum 60 trials. In the cue response task, the food was placed on a white flag whose position was randomly varied between the two goal arms to a total of 45 trials. The design ensures that the behavioral strategies suitable for solving each of the two tasks are mutually exclusive. $\boldsymbol{B}$, Two training conditions, space separate and time separate, were used. In the space separate condition, rats were trained/tested in both tasks during the same day, but each task was set in a dedicated context. On the fifth (last) day of retention testing, task-context pairings were swapped (context-task reversal). In the time separate condition, each rat was trained/tested in one context where it ran one task daily switching randomly between spatial and response learning across days. The two tasks were presented in random order.

ntations, is selectively dependent on a neural network centered on the hippocampus (HPC; Scoville and Milner, 1957; Packard et al., 1989; Squire and Zola-Morgan, 1991; McDonald and White, 1993; Eichenbaum, 2000; Aggleton and Pearce, 2001; White and McDonald, 2002), while habits, which include stimulus-response (S-R) behaviors, require an intact dorsolateral striatum (DSL; McDonald and White, 1994; Knowlton et al., 1996; Packard and McGaugh, 1996; Devan and White, 1999; Squire, 2004; Yin and Knowlton, 2004).

Recent data, however, have shown that the HPC and DSL can each support memories generally thought to be independent of these structures (Jacobson et al., 2012; O’Reilly et al., 2014; Ferbinteanu, 2016). In the most recent of these studies (Ferbinteanu, 2016), when rats concurrently learned a spatial navigation and a cue response task on the plus maze in the same context (understood here as the environment within which the animal behaves), lesions of the HPC and DSL no longer resulted in the expected double dissociation effects, which were still demonstrated if different groups of animals learned either of the two tasks alone. Instead, HPC and DSL lesions each impaired both spatial and response memory. Lesions of the dorsomedial striatum (DSM), a structure thought to be involved in flexible behavior or behavior based on action outcome associations (Ragozzino et al., 2002b; Yin et al., 2005; Lee et al., 2014), also impaired performance in both tasks, but in this case, the impairment occurred regardless of training parameters. These findings indicated that when spatial and response learning occur concurrently and in the same context, the HPC and DSL can be involved in behavior that is incongruous with their otherwise known functions. Thus, these structures can engage in functional coupling to integrate memories of different kinds. If HPC-DSL functional coupling is based on a nonspecific, general process that occurs in memory networks when distinct types of learning occur in temporal proximity or constant environment, then separating the learning experiences in either time or space would presumably reverse the coupling, and the behavioral contributions of the two memory structures would shift toward the typical dissociation present when the animals learn only one task at a time. To test this hypothesis, the current experiment evaluated the memory deficits caused by HPC, DSL, and DSM lesions when spatial and response learning were separated either in space but not time, or in time but not space (Fig. 1). One group of rats was trained in temporally adjacent spatial navigation and cue response tasks, but each task was learned in a dedicated context (a condition referred to below as "space separate"); this procedure separated the two kinds of learning in space but not time. A second group of rats learned the same tasks in one environment, but training on each task occurred during distinct days (a condition referred to below as "time separate"); this procedure separated the two kinds of learning in time but not space. After reaching a set 
performance criterion, animals received selective HPC, DSL, DSM, or sham lesions and were subsequently tested for retention following the same procedure as during training. Because rats trained in S-R tasks should be able to detect changes in context (McDonald et al., 2001), the contexts were swapped during the last day of testing in the space separate condition.

\section{Materials and Methods}

\section{Subjects}

Male Long-Evans rats (weight, 300-350 g; age, 4-6 months; Envigo) were individually housed ( $12 \mathrm{~h}$ light cycle) and tested during the day. The animals were acclimatized to the colony, randomly assigned to one of four groups (DSL lesion, HPC lesion, DSM lesion, or sham), and food was deprived to no more than $85-90 \%$ of ad libitum body weight and kept at this standard throughout the experimental procedure. A total of 63 animals (32 rats for the space separate condition and 31 rats for the time separate condition) were included in this study, but only data from animals with lesions restricted to the intended areas were incorporated in the final analysis, as follows: DSM and DSL, six rats/group in either training condition; HPC, seven rats/group in the space separate condition; and eight rats/group in the time separate condition. Sample size was determined based on previous work (Ferbinteanu, 2016) showing that the same type of lesions as in the current experiment resulted in large differences relative to control groups. After training, animals within each training condition were randomly assigned to one of the following four groups: sham; HPC lesion; DSL lesion; and DSM lesion. All procedures were approved by the SUNY Downstate Medical Center Animal Care Committee (protocol 15-10452). The investigator was not blinded to group allocation or training-testing procedures, or during data analysis.

\section{Apparatus}

The two plus mazes were made of gray polyvinyl chloride (PVC) and elevated $91 \mathrm{~cm}$ from the floor of two distinct rooms that each contained several visual cues and were illuminated distinctly. Each of the four arms was $61 \mathrm{~cm}$ long and $6.3 \mathrm{~cm}$ wide. A gray PVC block $(30.4 \mathrm{~cm}$ high, $6.3 \mathrm{~cm}$ wide, $15.2 \mathrm{~cm}$ deep) was used to block the start arm that was not in use for that trial. In each case, a rectangular waiting platform $(32 \times$ $42 \mathrm{~cm}$ ) was placed next to the maze. In the cued version of the task, a white visible flag also made of PVC was used to indicate the location of the food in the maze; during the spatial version of the task, the cue was placed on a table in the room and not in the maze.

\section{Experimental design: behavioral training and testing}

Experiment 1: learning two types of tasks in temporal proximity but in distinct contexts

One set of animals was trained in the two tasks on the same day, but each task was consistently associated with a dedicated environment. Each animal underwent $1 \mathrm{~d}$ of habituation, during which it was placed in each of the plus mazes in the presence of food; the visible flag was present in the maze in which the animal would then run the cue response task. Within each lesion group, the environments were counterbalanced across animals, and the tasks were learned in random order across days; once an animal completed a session in one environment, it was placed in the home cage and transferred to the second environment, where it underwent the second session. Training continued until the rats reached a criterion of $\leq 20 \%$ errors for 2 consecutive days in both tasks, after which they were assigned to one of the following four groups: HPC lesions, DSM lesions, DSL lesions, and sham controls. After a recovery interval of 5-10 d, retention was evaluated for 5 consecutive days. To test whether the animals associated a task with its dedicated environment, on the last day of postlesion testing, each animal performed each of the two tasks in the "other" environment.

Experiment 2: learning two types of tasks in the same context but at distinct times

A second group of animals was trained in the same two tasks, but in this case, each animal was trained in only one context and learned the two
Table 1. Lesion coordinates

\begin{tabular}{|c|c|}
\hline \multicolumn{2}{|l|}{ a. Hippocampal lesions } \\
\hline 1. AP, 3.1; L, $\pm 1.0 ; V, 3.6 ; 0.25 \mu \mathrm{l}$ & 6. AP, 5.0; L, $\pm 5.2 ; V, 5.0 ; 0.3 \mu \mathrm{l}$ \\
\hline 2. AP, 3.1; L, $\pm 2.0 ; V, 3.6 ; 0.25 \mu \mathrm{l}$ & 7. AP, 5.0; L, $\pm 5.2 ; V, 7.3 ; 0.3 \mu \mathrm{l}$ \\
\hline 3. AP, $4.1 ; L, \pm 2.0 ; V, 4.0 ; 0.25 \mu \mathrm{l}$ & 8. AP, 5.8; L, $\pm 4.4 ; V, 4.4 ; 0.3 \mu \mathrm{l}$ \\
\hline 4. AP, $4.1 ; L, \pm 3.5 ; V, 4.0 ; 0.25 \mu \mathrm{l}$ & 9. AP, 5.8; L, $\pm 5.1 ; V, 6.2 ; 0.40 \mu \mathrm{l}$ \\
\hline 5. AP, 5.0; L, $\pm 3.0 ; V, 4.1 ; 0.3 \mu \mathrm{l}$ & 10. AP, $5.8 ; \mathrm{L}, \pm 5.1 ; \mathrm{V}, 7.5 ; 0.40 \mu \mathrm{l}$ \\
\hline
\end{tabular}

b. DSM lesions DSL lesions

\begin{tabular}{ll}
\hline 1. $A P,+1.6 ; L, \pm 1.9 ; V, 4.80 .20 \mu l$ & 1. AP, $+1.6 ; L, \pm 3.0 ; V, 6.2 ; 0.20 \mu l$ \\
2. $A P,+0.5 ; L, \pm 2.2 ; V, 5.00 .20 \mu l$ & 2. AP, $+0.8 ; L, \pm 3.7 ; V, 6.6 ; 0.20 \mu l$ \\
3. $A P, 0.8 ; L, \pm 2.8 ; V, 3.60 .20 \mu l$ & 3. AP, $0.5 ; L, \pm 4.5 ; V, 6.6 ; 0.20 \mu l$ \\
\hline
\end{tabular}

AP, Anteroposterior from bregma; L, lateral from bregma; $V$, ventral from bregma. All coordinates are in millimeters.

tasks on different days. Task presentation was based on a pseudorandom sequence so that no task was performed more than $3 \mathrm{~d}$ in a row. An example of a training sequence across days is cue-cue-spatial-cue-spatial-cuespatial-cue-cue-spatial-spatial-spatial-cue-spatial-cue-spatial-cue-cue. The training continued until the animal reached the $\leq 20 \%$ error criterion on 2 consecutive days for each of the two tasks, after which the animals were randomly assigned to the four lesion subgroups. To avoid a context-specific effect, the same two environments as in experiment 1 were used in a counterbalanced manner (thus, in a lesion subgroup of six animals, three would be trained/tested in one environment and the remaining three in the other), as were the context/task of the first postlesion retention test. After a recovery interval of 5-10d, retention was evaluated for $10 \mathrm{~d}$ assigned randomly to five spatial and five response tests.

Spatial navigation and cue response tasks

All animals were pre-exposed to the maze in the presence of food for 2 consecutive days and then trained to walk from either the north or the south start arms to the end of west or east goal arms to obtain half a Fruit Loop. Between trials, the rats were placed on the side platform to wait for the next trial. Entry with all four paws into the unrewarded arm defined an error, which the rat was allowed to correct (thus, each trial was reinforced). Training procedures followed previously published protocols (Ferbinteanu, 2016) and used a spatial navigation task with serial reversals and a cue response task (Fig. 1). In both cases, the start and the goal arm were selected based on a pseudorandom sequence of 60 trials with at most three consecutive repetitions of the same type of journey (NE, NW, SE, or SW). On any given trial, the unused start arm was blocked and only one of the two goal arms was baited; thus, the apparatus functioned as a T-shaped maze that could be easily reversed across trials.

Spatial task. In the spatial task, the animal was rewarded for remembering spatial location. The position of the food was kept constant until the rat entered the correct goal arm in 9 of 10 consecutive trials. At that point, the other goal arm was baited and a new block of trials began. If the animal did not reach the criterion in a maximum of 15 trials, the location of the food was changed automatically, to avoid unbalanced reinforcement of any specific goal arm. Alternating trial blocks continued up to either four blocks or 60 total trials.

Cue task. In this case, the rats had to remember an association between the visible cue, whose location was rendered irrelevant by changing the start and goal positions based on a pseudorandom sequence, and a motor response, which was the walk toward the cue. Animals received 45 trials in each session, a number approximately equal to the number of trials necessary to complete four blocks of trials in the spatial task. Thus, each goal arm was approximately equally rewarded both within and across tasks.

\section{Lesions}

At the end of the training phase, animals were randomly assigned to one of the following four groups: sham control group, HPC lesion group, DSL lesion group, and DSM lesion group. Rats were anesthetized with 
isoflurane and diazepam (10 mg/kg). Atropine ( $5 \mathrm{mg} / \mathrm{kg}$ body weight) was also administered to avoid fluid accumulation in the respiratory tract. Neurotoxic lesions were made by injecting either a solution of $5 \mathrm{mg} / \mathrm{ml}$ NMDA in phosphate buffer, $\mathrm{pH} 7.4$ (Tocris Bioscience), or a solution of quinolinic acid (Tocris Bioscience; $25 \mathrm{mg} / \mathrm{ml}$ in phosphate buffer titrated with sodium hydroxide to $\mathrm{pH}$ 7.4) through a 30 gauge cannula attached to a minipump $(0.2 \mu \mathrm{l} / \mathrm{min}$; Model NE-4000, New Era Pump Systems). At the end of each injection, the cannula was left in place for $3 \mathrm{~min}$, retracted $0.5 \mathrm{~mm}$, and left in this location for $2 \mathrm{~min}$, after which it was slowly and completely retracted. The coordinates of each injection and the volumes injected are presented in Table 1 . To prevent seizure development, a second intraperitoneal injection of diazepam ( $10 \mathrm{mg} / \mathrm{kg}$ body weight) was administered before neurotoxin infusion, and the animals were monitored until completely awake and active in their home cages. Sham animals were anesthetized, incised, and sutured.

\section{Statistical analysis of behavioral data}

Percent performance error was calculated for each rat during each day of testing, and a mean was calculated for each group during each day. All analyses were performed using SAS version 9.2 (SAS Institute). Differences in performance were assessed using two-way mixed-model analyses that included data from all animals for each of the two training conditions. Time (in days) and lesion group were entered into the model as categorical independent variables, and performance as the percentage error on repeated time points was entered as a dependent variable. The degrees of freedom were computed according to the Satterthwaite formula, which takes into consideration the variance within the group along with the sample size and is robust against heterogeneity of variance. Where overall analyses indicated significant differences, differences in performance between each lesion group and the sham group were further investigated. The effects of the reversal test (fifth postlesion day in the space separate condition) were assessed by using a two-tailed paired $t$ test to evaluate for each group the behavioral performance during the test relative to the performance during the previous day.

\section{Tissue preparation and processing for histology}

Rats were overdosed with isoflurane (administered in a closed environment) and perfused transcardially with normal saline followed by $10 \%$ formalin for tissue fixation. Coronal sections $(40 \mu \mathrm{m})$ were cut on a cryostat and stained with cresyl violet to evaluate the extent of the lesion.

\section{Lesion assessment}

Brains were coronally sectioned. Each fourth section in the striatum lesion groups, and each fifth section in the hippocampal lesion groups was mounted on a microscope slide to be used for lesion evaluation. We first visually inspected the sections under the microscope and traced each lesion on a set of histologic plates (Paxinos and Watson, 1988). Data from animals whose lesions were not sufficiently inclusive (i.e., not encompassing most of the targeted area) and selective (i.e., extending bilaterally to significant portions of other brain areas) were excluded from further analysis. The lesions that met the criteria underwent quantification analysis. All slides incorporated in the analysis were scanned using a slide scanner (Aperio AT2, Leica Biosystems) to generate digital images of the sections. Dedicated software (ImageScope, Leica Biosystems) was then used to visualize and measure for each section the area of preserved hippocampal or striatal tissue. This procedure was also performed for brain tissue from four control animals for the dorsal striatum and four control animals for the hippocampus. Ten sections were selected from each animal in the DSM and DSL groups and 14 sections in the HPC groups (approximately half the total number of sections) so that approximately equivalent levels on the anteroposterior axis were captured in the analysis across animals. The values of the preserved tissue areas were then summed to estimate the total healthy tissue for each animal. (Because the brain tissue was sampled at regular intervals of $200 \mu \mathrm{m}$ for all HPC tissue and $160 \mu \mathrm{m}$ for all striatal tissue in control and lesion animals alike, multiplying by the constant distance between sections was not necessary to obtain an estimate of the lesion size as a percentage of the total structure.) The totals for the control animals were averaged for the dorsal striatum and hippocampus, and the resulting values were considered as $100 \%$ in the computation that evaluated the lesion size. For each lesioned animal, the percentage of healthy tissue was computed by dividing the area of healthy tissue in their brain by the corresponding area in the control brain; the percentage of lesion was then obtained as the difference between the percentage of healthy tissue and $100 \%$. For each lesion group, we compared the percentage of damage across the three experimental conditions using an unpaired twotailed $t$ test (SAS Institute).

\section{Results}

\section{Lesions encompassed the intended brain areas}

For each animal, the lesion's selectivity was evaluated; only data from animals with damage restricted to the intended brain areas were considered for further analysis. Neurotoxic cortical damage was small and typically only the mechanical damage at the cannula insertion point was present. All tissue with signs of gliosis was considered lesioned, regardless of whether the principal neurons might have been spared at the periphery of the affected area (Fig. 2A, orange arrowheads). For the HPC lesion groups, all areas of HPC proper (dentate gyrus, CA3, CA2, and CA1) incurred large, almost complete, damage in both dorsal and ventral portions of the structure (Fig. 2A, top two rows). In general, the ventral tip of the HPC was spared, as well as the ventral blade of the dentate gyrus at the most anterior HPC pole. There was minor damage to the subiculum in the anterior areas, but no damage was found in the entorhinal cortex or other nearby cortical regions. Two animals in each of the two HPC lesion groups had small cortical damage. There was small unilateral damage in the thalamus in one rat in the time separate condition resulting from a deep penetration of the cannula, which was not accompanied by neurotoxic damage; the data of this animal did not show marked differences from the data of the rest of the subjects in the same group. Regarding the dorsal striatum, in the rat there is no physical separation between the functionally distinct medial and lateral areas, which are histologically homogeneous. The distinct functional roles of these areas (acquisition of flexible, goaldirected, outcome-sensitive associations for DSM vs rigid, goalindependent, outcome-insensitive instrumental responses for DSL) are related to their cortical input, which originates in various prefrontal areas and the somatosensory cortex, respectively (Voorn et al., 2004). There is also some overlap between the inputs as orbitofrontal, anterior cingulate, and precentral cortices reach the DSL as well as the DSM (Mailly et al., 2013, their Fig. 4). In this study, the aim was to achieve DSM and DSL lesions as inclusive as possible that would conform to known topography of anatomic connections (McGeorge and Faull, 1989; Voorn et al., 2004; Groenewegen, Voorn, and Scheel-Krüger, 2016). For DSL lesion groups, three animals in the space separate condition and four animals in the time separate condition had small unilateral damage to claustrum, endopiriform cortex, lateral orbital cortex, and globus pallidus. For DSM lesion groups, one animal in the space separate condition and two animals in the time separate condition had small damage to globus pallidus. Overall, the lesions distinguished well between the medial and lateral areas of the dorsal striatum with overlap restricted to the most anterior, dorsal, and lateral regions of the striatum (Fig. $2 B$ ). Lesion size was not significantly different across training conditions in any of the lesion groups (DSM: $t_{(10)}=1.678, p=0.1241$; DSL: $t_{(10)}=$ $0.166, p=0.8711$; HPC: $t_{(13)}=1.073, p=0.3084$; Fig. $2 A$ right inset, $B$ ).

Basic aspects of behavior were not affected by the lesion. Animals in all lesion groups continued to readily enter the goal arms from the central stem, were highly motivated, and 
A

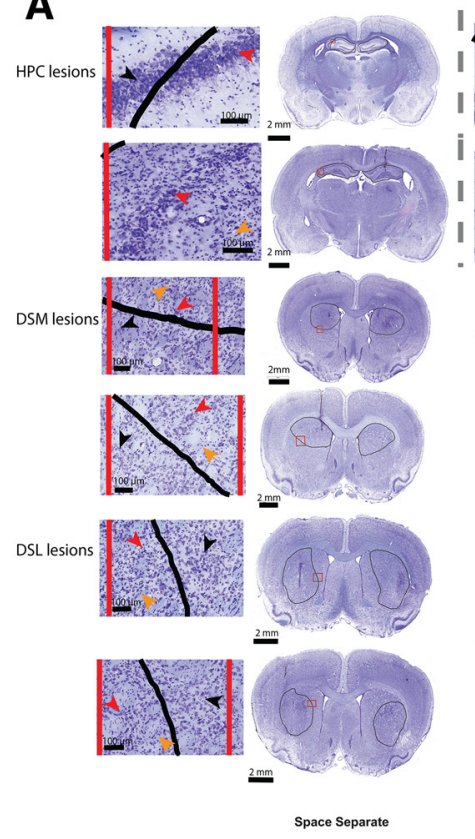

B

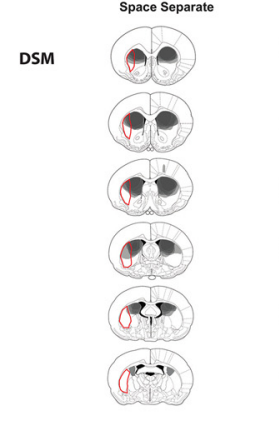

DSL

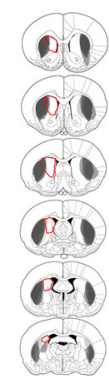

HPC

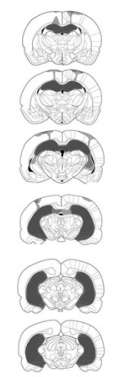

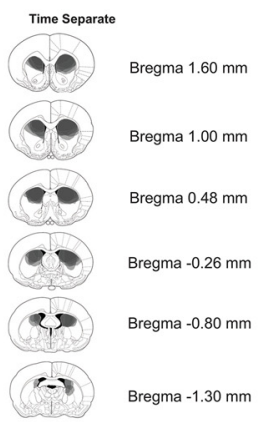
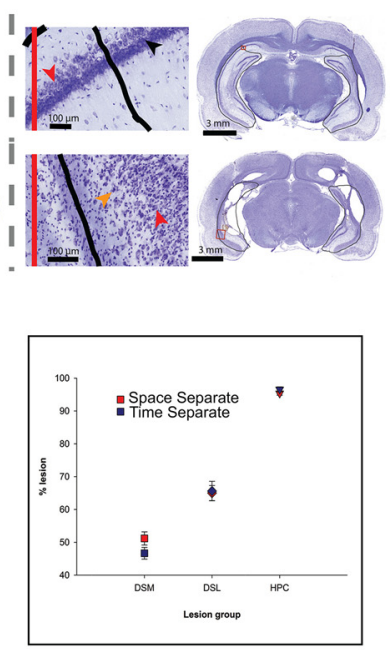

Bregma $-1.30 \mathrm{~mm}$

Bregma $1.60 \mathrm{~mm}$

Bregma $1.00 \mathrm{~mm}$

Bregma $0.48 \mathrm{~mm}$

Bregma $-0.26 \mathrm{~mm}$

Bregma $-0.80 \mathrm{~mm}$

Bregma $-1.30 \mathrm{~mm}$

Bregma - $2.30 \mathrm{~mm}$

Bregma $-3.14 \mathrm{~mm}$

Bregma $-4.40 \mathrm{~mm}$

Bregma $-5.20 \mathrm{~mm}$

Bregma $-5.60 \mathrm{~mm}$

Bregma $-6.04 \mathrm{~mm}$

Figure 2. Lesions were localized in the targeted areas. $\boldsymbol{A}$, Each of the six lesion groups (HPC, $n=7$ and $n=8$; DSM, $n=6$ and $n=6$; and DSL $n=6$ and $n=6$; in space separate and time separate training conditions) is represented by one lesion illustration. The top two rows (two different rats) show sections in dorsal (left) and ventral (right) HPC from the same animal. Photomicrographs of coronal sections through the brain at low magnification indicate the lesion area (circled in black). Enlarged parts of the small area within the red square are presented to the left in each case to enable comparison between degenerating or dead cells (red arrowheads) and healthy cells (black arrowheads). For both DSM and DSL groups, tissue with any signs of gliosis (e.g., astrocytic invasion among apparently normal cells, orange

completed entire sets of trials for each type of task. There was also no indication that animals with HPC lesions would adopt a body-turn strategy (presumably dependent on the DSL) or that the animals with DSL lesions would persevere in going to one spatial location.

\section{DSL supported spatial memories acquired in the same environment as response memories; HPC supported response memories acquired concurrently with spatial memories}

Rats with DSL lesions showed a significant spatial memory deficit relative to controls throughout the $5 \mathrm{~d}$ of retention testing when spatial and response memories were acquired in the same environment (Fig. 3A, left, Table 2, a). Directly comparing the spatial performance of animals with this type of lesion in the two training conditions highlighted the large difference that the training procedure caused on performance $\left(F_{(1,47.9)}=84.14\right.$, $p<0.00,001$; Fig. $3 B$, top left). At the same time, as expected, DSL lesions impaired response memory regardless of training procedure (Fig. $3 A$, right, Table 2 , b; for more on the recovery from the memory deficit in the space separate condition, see below). Thus, while DSL supports a rigid habitual response to a single cue in general, it can also become involved in a behavior illustrative of declarative memory if spatial and response learning occur in the same environment. Current data do not clarify whether the DSL formed its own context representation, directly accessed the context representation formed by the HPC, or accessed the HPC context representation at the level of control over the motor output. The results of the reversal test in the space separate condition suggested that in this experiment, context representations were selectively dependent on the HPC because, unlike for the rest of the animals, the performance of this lesion group was not altered by the context swap in either of the two tasks (Fig. 3A, top row, Table 3).

The performance of the HPC lesion groups on the cue response task showed a pattern of impairment largely complementary to the one described above for animals with DSL lesions on the spatial task (Fig. $3 A$, right). Specifically, these groups were consistently impaired in response behavior when the rats formed spatial and response memories in close temporal succession but in distinct environments (Fig. 3B, second row right, Table 2, c). In the other training condition, when spatial and response memories were acquired in the same environment across different days, the HPC lesion group had a deficit in response memory only on the first day of retention testing. The result was caused by the poor performance of three out of eight animals (Fig. $3 B$, inset of second row, right-hand panel). The rapid recovery of the memory deficit of these animals on the subsequent day suggested that an already existing cue-response association regained control over the motor output. Directly comparing response

arrowheads) was marked as lesioned. Similarly, HPC lesions included areas where neurons were either missing or degenerating, or astrocytes were seen in large numbers. The inset presents the results of the quantification analysis. For the DSM and DSL lesions, proportions of lesions were calculated based on the volume of the entire dorsal striatum. $\boldsymbol{B}$, Extent of the lesions. For all animals whose data were included in the analysis, lesioned areas were marked and superimposed on the same set of corresponding diagrams. Areas lesioned in all animals of a group show as the darkest gray, and areas lesioned in only one animal are shown as lightest gray. The brain tissue was damaged at the insertion point of the injection needle in all cases; the figure shows only damage larger than what would be expected from the 30 ga cannula. The red contours show the approximate area of the intended DSL lesion superimposed on the DSM lesions and vice versa (adapted from Groenewegen et al., 2016). 
A

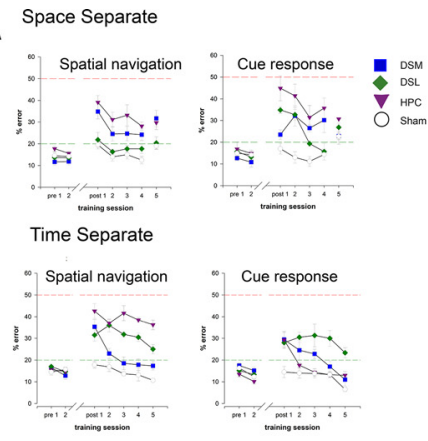

B

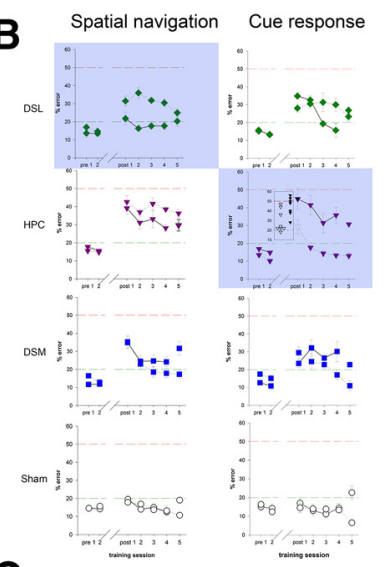

C Spatial navigation Cue response
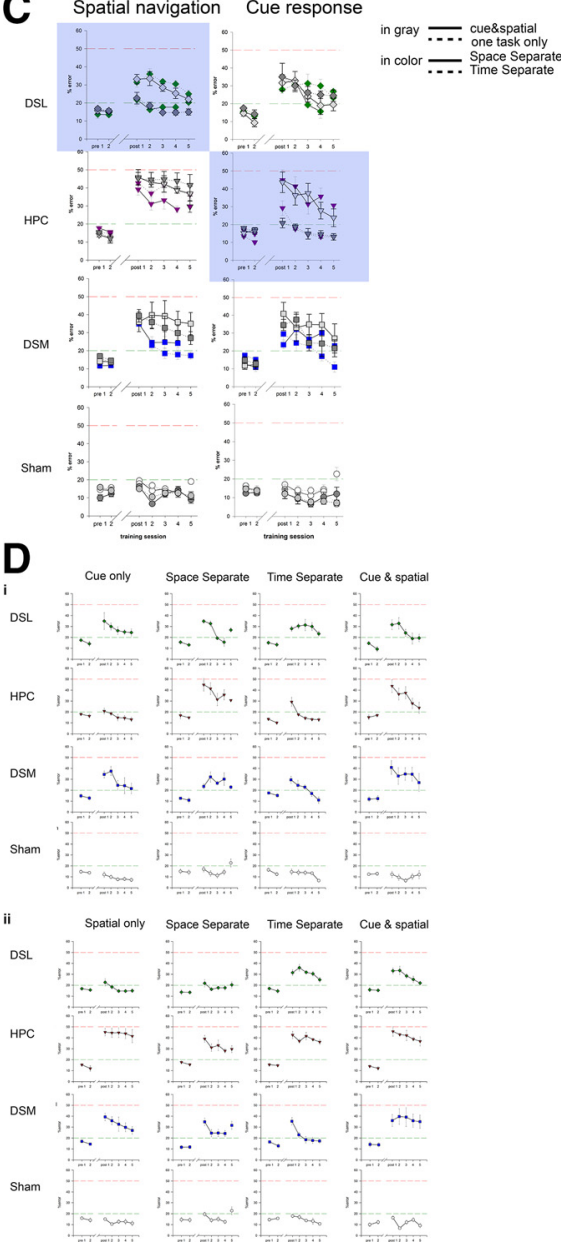

Figure 3. Behavioral performance for all groups in the two training conditions. $\boldsymbol{A}$, Data organized to facilitate comparisons among lesion groups within the same training condition and during the reversal test in the space separate condition. All rats performed well before performance in animals with HPC lesions that underwent different training procedures underscored the large differences in the behavior of these two groups $\left(F_{(1,60)}=114.55, p<0.0001\right)$. In contrast with the selective effect on response performance, HPC lesions consistently impaired spatial navigation (Fig. 3B, second row left, Table 2, d). Thus, the HPC is critical for spatial representations in general, but it can additionally support response memories if they are formed in temporal proximity to spatial memories.

\section{DSM supported flexible behavior}

Although the DSL and DSM are both parts of the dorsal striatum, the DSM is involved not in habitual, but flexible, goaldirected behaviors (Ragozzino et al., 2002a,b; Yin et al., 2005, 2006; Gremel and Costa, 2013). The current results were in agreement with this idea, as DSM lesions generally impaired performance regardless of training conditions in both the spatial

the surgery, and sham animals continued to do so after the surgery. In contrast, lesioned animals exhibited memory deficits. In the space separate condition, the DSL group had normal spatial and impaired response memories while the HPC group made many errors in both spatial and response tasks. In the time separate condition, the pattern reversed: the DSL group showed both spatial and response memory deficits, while the HPC group had impaired spatial but largely normal response memory (see $\boldsymbol{B}$ and text for details of performance during the first day postlesion). The DSM groups showed various degrees of memory deficits throughout retention testing. Context reversal disrupted the performance of sham animals. Rats with HPC lesions did not alter their performance, the DSL lesion group performed worse in the response task, and the DSM lesion group performed worse in the spatial task. For all graphs, vertical axes show the percentage error in behavioral performance (mean \pm SEM), and horizontal axes show training/testing sessions, with the break marking the surgery point. The green horizontal lines at 20\% indicate the criterion threshold. The red horizontal lines at $50 \%$ indicate chance performance level. $\boldsymbol{B}$, Same data as in $\boldsymbol{A}$ organized to reveal effects of training conditions. DSL was critically involved in response memory overall, but also supported spatial navigation when spatial and response learning occurred in the same context during different days (top row, left). HPC was critical for spatial navigation overall, but additionally supported cue response when spatial and response learning occurred concurrently in different contexts (second row, right). The inset shows individual data points during the first day of retention testing for the space separate (black) and time separate (white) conditions. Five of the eight animals in the time separate condition were close to criterion level. In contrast, six of seven animals in the space separate condition showed severe impairment. Sham animals performed well regardless of training paradigm. In all cases, statistical analyses included only data from days 1-4 postlesion training (i.e., excluded the context/task reversal test). Blue highlights indicate the datasets that do not conform to the current thinking on memory organization. $\boldsymbol{C}$, Same data as in $\boldsymbol{A}$ and $\boldsymbol{B}$ (in color), combined with previous data (in gray; Ferbinteanu, 2016) illustrating the effects of DSL, HPC, and DSM lesions on spatial and response memories after training in only one of the two tasks (one task only, dark gray) or after training in the two tasks concurrently in the same context (Cue response and Spatial navigation, light gray). The effects of training paradigm on the performances of DSL groups in spatial navigation and HPC groups in cue response are similar in the two experiments (blue highlights). Thus, the time factor can account by itself for the effect on response memory induced by HPC lesions. Similarly, the space factor can account by itself for the effect on spatial memory caused by DSL lesions. In contrast, the performances of DSL lesion groups in the cue response task and HPC lesion groups in the spatial navigation task varied between this and previous experiments, as did the performance of the DSM lesion groups overall. The behavior of sham animals remained consistent across training protocols. $\boldsymbol{D}$, Same data as in $\boldsymbol{C}$ replotted to facilitate visualizing the distinct contributions DSL, HPC, and DSM had to normal behavior. The training protocol is indicated at the top of each column, and the performance of the sham group in that condition is shown in the bottom panel. Comparing across the bottom row reveals that normal animals performed similarly regardless of training. However, in each case the performance was supported to different degrees by the three brain areas, an effect modulated by the training protocol. $\boldsymbol{i}$, Cue response. ii, Spatial navigation. 
Table 2. Response and spatial memory deficits in DSL and HPC lesion groups, respectively

\begin{tabular}{|c|c|c|c|c|c|c|c|c|}
\hline & Space separate & DF & $t$ Value & $\operatorname{Pr}>|t|$ & Time separate & DF & $t$ Value & $\operatorname{Pr}>|t|$ \\
\hline \multirow[t]{4}{*}{ a. DSL vs sham spatial task } & Day 1 post & 58.5 & 0.62 & 0.5377 & Day 1 post & 93.3 & 4.18 & $<0.0001$ \\
\hline & Day 2 post & 58.5 & 0.62 & 0.5377 & Day 2 post & 93.3 & 5.87 & $<0.0001$ \\
\hline & Day 3 post & 58.5 & 0.71 & 0.4814 & Day 3 post & 93.3 & 5.56 & $<0.0001$ \\
\hline & & & & & Day 5 post & 93.3 & 4.39 & $<0.0001$ \\
\hline \multirow[t]{3}{*}{ b. DSL vs sham cue response task } & Day 1 post & 84.1 & 4.22 & $<0.0001$ & Day 1 post & 130 & 3.7 & 0.0003 \\
\hline & Day 2 post & 84.1 & 4.65 & $<0.0001$ & Day 2 post & 130 & 4.52 & $<0.0001$ \\
\hline & & & & & Day 5 post & 130 & 4.61 & $<0.0001$ \\
\hline \multirow[t]{5}{*}{ c. HPC vs sham cue response task } & Day 1 post & 84.1 & 6.84 & $<0.0001$ & Day 1 post & 130 & 4.32 & $<0.0001$ \\
\hline & Day 2 post & 84.1 & 6.98 & $<0.0001$ & Day 2 post & 130 & 1.06 & 0.2903 \\
\hline & Day 3 post & 84.1 & 4.97 & $<0.0001$ & Day 3 post & 130 & 0.16 & 0.8742 \\
\hline & Day 4 post & 84.1 & 5.25 & $<0.0001$ & Day 4 post & 130 & -0.02 & 0.9806 \\
\hline & & & & & Day 5 post & 130 & 1.9 & 0.0592 \\
\hline d. HPC vs sham spatial task & & & & & Day 5 post & 93.3 & 8.37 & $<0.0001$ \\
\hline \multirow[t]{5}{*}{ e. DSM vs sham spatial task } & Day 1 post & 58.5 & 4.07 & 0.0001 & Day 1 post & 93.3 & 5.36 & $<0.0001$ \\
\hline & Day 2 post & 58.5 & 2.79 & 0.0071 & Day 2 post & 93.3 & 1.89 & 0.0621 \\
\hline & Day 3 post & 58.5 & 2.57 & 0.0128 & Day 3 post & 93.3 & 1.48 & 0.1422 \\
\hline & Day 4 post & 58.5 & 3.1 & 0.003 & Day 4 post & 93.3 & 1.43 & 0.1563 \\
\hline & & & & & Day 5 post & 93.3 & 2.04 & 0.044 \\
\hline \multirow[t]{5}{*}{ DSM vs sham cue task } & Day 1 post & 84.1 & 1.54 & 0.128 & Day 1 post & 130 & 4.11 & $<0.0001$ \\
\hline & Day 2 post & 84.1 & 4.53 & $<0.0001$ & Day 2 post & 130 & 2.88 & 0.0047 \\
\hline & Day 3 post & 84.1 & 3.63 & 0.0005 & Day 3 post & 130 & 2.47 & 0.015 \\
\hline & Day 4 post & 84.1 & 3.74 & 0.0003 & Day 4 post & 130 & 1 & 0.317 \\
\hline & & & & & Day 5 post & 130 & 1.23 & 0.2199 \\
\hline
\end{tabular}

Each section lists the results of $t$ tests comparing performance of animals with DSL, HPC, or DSM lesions to the performance of corresponding sham groups. The tests were planned a priori and run after the results of the two-way mixed analyses indicated highly significant main effects and interactions; the degrees of freedom were computed based on Welch-Satterthwaite equation. Post, After lesioning.

Table 3. Results of the reversal test

\begin{tabular}{lllll}
\hline & Lesion & DF & $t$ Value & $\operatorname{Pr}>|t|$ \\
\hline \multirow{4}{*}{ Spatial task } & DSL & 25 & -1 & 0.3259 \\
& DSM & 25 & -2.82 & 0.0093 \\
& HPC & 25 & -0.64 & 0.5293 \\
\multirow{4}{*}{ Cue task } & Sham & 25 & -2.44 & 0.022 \\
& DSL & 25 & -2.44 & 0.022 \\
& DSM & 25 & 1.6 & 0.1212 \\
& HPC & 25 & 1.18 & 0.2485 \\
& Sham & 25 & -1.82 & 0.0802 \\
\hline
\end{tabular}

The table list the results of a priori planned $t$ tests comparing for each lesion group the performance during the reversal test in the space separate condition relative to the preceding day.

and response tasks. The degree of impairment varied with training procedure, but the size of the effect was considerably smaller than for those of the other two types of lesions and stronger in the response than in the spatial task (Fig. $3 A, B$, third row; spatial navigation: $F_{(1,47.9)}=3.57, p=0.0650$; cue response: $F_{(1,47.9)}=$ $5.11, p=0.0283$; Table $2, e)$. This pattern of results is not surprising given that in each task, the animals have to adapt the body turn at the center of the maze to their start position relative to the goal. The data also suggested that the DSM lesions may cause slightly more impairment in the space separate condition overall, which is also not surprising because, in this case, the animals have to switch from one task to another within the same test session. Thus, the DSM supported both spatial navigation and habitual response to a discrete cue, and overall the data indicate that its contribution is consistent with a role in behavioral flexibility.
The magnitudes of spatial and response memory deficits were modulated by training procedure

A large body of empirical data links the DSL to S-R behavior and the HPC to spatial navigation. The results of the current experiment confirmed this idea but also indicated that for both structures, the magnitude of the memory deficit was modulated by the training condition (HPC: $F_{(1,59.9)}=9.86, p=0.0026$; DSL: $F_{(1,47.3)}=4.54, p=0.0383$; Fig. $3 B$, top row right and second row left). Animals with HPC lesions had a permanent spatial memory deficit regardless of how the task was learned. Animals with DSL lesions showed permanent impairment in response memory in the time separate condition. In the space separate condition, this lesion group initially showed a strong impairment during the first $2 \mathrm{~d}$ of retention testing, which then declined rapidly and was eliminated in the next $2 \mathrm{~d}$. Thus, in this case and in this case only, the remaining neural circuits (which do not include remnants of the DSL network) can rescue the response behavior. As already described above, training condition also modulated the performance of the DSM lesion groups but note that in this case, the space separate condition was consistently associated with more impairment, which was not the case with DSL and HPC lesions. In contrast, the training condition did not affect the proficient performance of normal animals (Fig. 3B, bottom row). Collectively, these findings showed that the specialized representations formed by each of the three brain structures forms can support the corresponding behaviors as a matter of degree, depending on training condition. If so, then it is of interest to know how the results from the current experiment compare to previous work (Ferbinteanu, 2016), when animals with similar types of lesions learned the same two tasks concurrently and in 
the same environment (i.e., in a way that combined the spatial and temporal factors). Thus, the past and current results were plotted on the same graphs (Fig. 3C). Comparison between these two datasets revealed two facts. First, for both the DSL and HPC, the pattern of contribution to the task incongruous with the style of information processing of that structure-DSL to spatial navigation and HPC to cue response-were remarkably similar (Fig. $3 C$, blue highlights). Because the DSL groups performed the same in spatial navigation regardless of whether the rats learned the two tasks concurrently (previous data) or on distinct days (current data), it seems that the temporal factor did not modulate the ability of the DSL to integrate distinct memories based on space. Similarly, because the HPC groups performed the same on cue response regardless of whether the rats learned the two tasks in the same (previous data) or distinct (current data) contexts, it seems that the spatial factor did not impact the ability of the HPC to integrate distinct memories based on time. This finding supports the idea that the HPC and DSL integrate memories based not only on distinct, but also largely complementary functional principles. Second, the comparison between past and present results further confirmed that in tasks congruous with the type of representation that each brain area forms, the lesion effects were modulated by the training protocol (Fig. 3C). The effects do not suggest a hierarchical organization such that the highest levels of deficit would result when spatial and temporal factors are combined (animals learn cue and spatial tasks together in the same context; the previous experiment), next down would be the effects corresponding to one of the two factors (temporal or spatial by themselves; the present experiment), and the least deficit would result when neither factor is present (animals learn only one task at a time). Thus, depending on learning circumstances, the same normal memory-based behavior (Fig. $3 \mathrm{Di}, \mathrm{ii}$, bottom two rows), currently thought of as being guided by a set neurobiological basis, can be in fact supported by vastly different neural networks, which can extend across multiple core memory structures. Thus, even these two simple tasks reveal that behaviors are highly degenerate.

\section{Alternative explanations of current results \\ Effects of consecutive behavioral tasks}

The current results indicate that rats with HPC lesions perform poorly in the response task when they learn the two tasks successively in different environments (space separate condition) and rats with DSL lesions perform poorly in the spatial task when they learn the two tasks in the same environment during different days (time separate condition). One potential cause of poor performance in these cases may be an interference effect whereby unsuccessful prior engagement in one task would cause increased error rate during a subsequent test. The design of the experiment controlled for an order effect by using a counterbalancing procedure, but this possibility was nonetheless explored by separating the results based on task order: HPC lesion group averages day 1-day 5: percentage error spatial-to-cue: 42.33, 40.25, 30.33, 34.66 , and $25.5 \%$; versus percentage error cue-to-spatial: 46.75 , $43.00,32.25,42.00$, and $37.66 \%$; DSL lesion group averages day 1-day 5: percentage error cue-to-spatial: 33.25, 37.66, 32.24. 35.0, and $21.66 \%$; versus percentage error spatial-to-cue: $28.00,34.33$, $29.00,28.25$, and $28.33 \%$. Even as the small number of points in this dataset precludes meaningful formal statistical comparisons between these levels of performance, the results show that in both lesion groups there is a performance deficit regardless of the order of task presentation (all numbers are larger than the $20 \%$ error criterion). Moreover, when animals with HPC lesions ran the spatial task first, they made fewer-rather than moreerrors. Thus, the order in which the animals perform the two tasks may generate complex interactions between hippocampal and striatal memory systems that bridge intervals from minutes to days, but the current results indicate that such interactions may at most modulate the lesion effects rather than cause them.

\section{Alternative strategies}

Because the current behavioral tests restricted the behavior of animals to right and left turns, it is possible that the response deficit in HPC animals and spatial deficit in the DSL animals were related to the adoption of inadequate behavioral strategies. Specifically, HPC animals may have adopted a body turn strategy during response tasks and, correspondingly, DSL animals may have adopted a spatial strategy during spatial tasks. Such a possibility is unlikely, however, because in the current paradigm, the response and spatial strategies are mutually exclusive and the choice of the "wrong" strategy is never reinforced. Indeed, analysis of the preferred body turns recorded during the first day of retention testing during the response task in HPC animals did not reveal significant differences between HPC and sham groups whether the turns were considered overall, or only during the error trials (all trials: $t_{(11)}=2.067, p=0.063$; error trials only: $t_{(11)}$ $=1.096, p=0.296$ ). The presence of a preferred body turn during a given test session could not be considered a reflection of a behavioral/psychological phenomenon because the trial sequence (computed as a random sequence of four different journeys with the condition of no more than three journeys of the same kind in a row) did not divide equally between right and left turns (analysis of a sample of these sequences showed the following percentage right/percentage left turns: 45\%/55\%; 60\%/40\%; 48.88\%/ $51.11 \%$; 53.33\%/46.67\%; 35.55\%/64.44\%; 44.54\%/55.55\%; and $48.88 \% / 51.11 \%$, respectively; the rats do not develop a bias in response because for any given animal, right and left turns balance out across training and testing days). Similarly, there was no evidence that animals with DSL lesions would adopt a "hyperspatial" strategy during spatial tests. Such a strategy would be reflected either by many errors massed after the switch of the goal locations or by exclusive response to only one of the two spatial locations. However, animals with DSL lesions reversed the goal location, but then proceeded to make errors that were distributed throughout the trial block (Extended Data Fig. 3-1). Thus, performance deficits in the response and spatial tasks occurring after HPC and DSL lesions, respectively, cannot be attributed to adoption of an alternative inadequate behavioral strategy.

\section{Stereotypical behavior}

A third possibility is that the behavior of lesioned animals would become increasingly stereotypic as retention testing would progress. For example, rats would run at increasingly higher speed through the trials. Such a change occurred but was limited to the initial stages of training phase, when the animals acquired the basic rule of the tasks (walk from the start arm to the end of one of the goal arms to obtain food); neither type of lesion introduced modifications in the general aspects of behavior. Moreover, if behavior became increasingly stereotypic during retention testing, one would expect that that the level of impairment should remain consistent across days. In contrast, the data show some degree of recovery from the memory deficits for both HPC and DSL lesion groups. The factor that seemed to affect the speed of movement involved individual differences: some animals were more motivated, less anxious, and more able to focus than 
others. To counteract any potential effects of this factor, rats were randomly assigned to the lesion groups.

\section{Discussion}

Patient H.M.'s selective amnesia that followed after the bilateral resection of his medial temporal lobes (Scoville and Milner, 1957) indicated that severe episodic memory impairment could result from a focal brain lesion. Instead, the pattern of memory deficits documented in H.M. and similar patients showed that localized neural circuits are responsible for different kinds of memories. This discovery triggered numerous subsequent studies in which the mnemonic functions of various brain areas were extensively investigated. The analytical approach provided much information about memory organization in the brain and the underlying mechanisms. However, the opposite problem, of how different memories are integrated, remains poorly understood. The current data contribute to filling some of this gap, as they demonstrate that the DSL and HPC integrate different types of memories based on spatial and temporal factors, respectively. Notably, the memories involved were of distinct kinds, suggesting a real possibility that activity in one memory circuit may affect processes in a different memory circuit (Kim and Baxter, 2001). Despite the simplicity of the experimental manipulations, the implications of these results are far reaching and lead to significant questioning of our current perspective on memory architecture.

The ability of the HPC circuits to integrate different types of memories, primarily by using temporal rather than spatial factors, indicates that the "when" of episodic memory may act as the thread linking the "where" (and possibly also the "what") of individual events, a prospect also suggested by recent data (Mankin et al., 2015; Rubin et al., 2015; Cai et al., 2016). Thus, the HPC is critical for spatial representations in general, but it can additionally support response memories if they are formed in close temporal proximity to spatial memories. This property of the HPC network, which does not extend to context, dovetails well with the quintessence of episodic memory. We recollect as a coherent unit events that occur in close succession, regardless of environment, and distinguish among events that happen in the same space at different points in time. If cognitive memories can be thoroughly integrated with habits within the HPC, then it is not surprising that habits, which can be performed without conscious awareness (as for example in typing) can also be consciously recollected.

A second effect of a memory architecture in which HPC can support habits is that a habit can be rescued after damage to the neural network primarily responsible for its acquisition (i.e., DSL), as the current data indicate that it may indeed be the case. When animals acquired the response and spatial memories in temporal juxtaposition and HPC was involved in response memory, DSL lesions caused a transient rather than permanent deficit in response memory (Fig. 3Di, second and fourth panels, first two rows). In contrast, when the response memory was formed either by itself or in temporal separation from the spatial memory, both cases in which the HPC network did not contribute to the behavior, the impairment was long lasting (Fig. 3Di, first and third panels, first two rows). This fact also means that the persistence of marked impairment in the response task after DSL lesions in the time separate condition (Fig. 3Di, third panel of the first row) does not reflect a "weaker" response memory trace following training spaced across days. Thus, these data strongly suggest that HPC can independently support S-R behavior. (This analysis, however, should not be interpreted as implying that HPC and DSL contributions to behavior combine in linear fashion.)

The complementary aspect of the functional role of DSL revealed by the current data is that while DSL supports a rigid habitual response to a single cue in general, it can also become involved in a behavior illustrative of cognitive memory if the two types of learning occur in the same space. This property of the DSL network may be involved in drug relapse, whereby exposure to an environment previously associated with drug abuse (an extreme form of habit) leads to renewed drug consumption. Even as the results of the reversal test in the space separate condition suggested that context representations were selectively dependent on the HPC, reports that context memories can become HPC independent (Lehmann et al., 2009; Sparks et al., 2011) and that the DSL can be involved in context memories (White and Salinas, 2003) leave open the possibility that along with the HPC, the DSL may also form its own context representation. The precise neural processes than enable DSL to support spatial and response memories acquired in the same environment remain currently unknown, although they may be related to its functional heterogeneity (Vicente et al., 2016) and to the dynamic reorganization of the DSM-DSL circuits during skill learning (Yin et al., 2009). Whatever contextual representation DSL may form, it is, however, insufficient to support spatial navigation on its own, a result in agreement with the permanent spatial deficit seen in animals with HPC lesions that have undergone extensive spatial training (Clark et al., 2005). The asymmetry between the capabilities of the two structures relative to behavior may have an evolutionary basis: DSL and HPC evolved at different points in time during phylogeny and, thus, the HPC built on an already existent DSL network (Murray et al., 2016). Collectively, these findings have important implications for addiction. If the HPC network can independently support a habit while the DSL network can use context to integrate habits and cognitive memories, then treating addiction has to address simultaneously striatal and hippocampal processes from the perspective that these two networks operate as an integrated unit rather than as separate modules, each with a distinct contribution along a set function (Ferbinteanu, 2019).

The interplay between HPC and DSL networks in supporting behavior likely involves the DSM and its connections to prefrontal areas (specifically, orbitofrontal and prelimbic cortices; Sharpe et al., 2019), which are known to be involved in decisionmaking in both animals and humans (Fellows, 2018). As predicted by a role in behavioral flexibility, DSM supported both spatial and response memories regardless of training condition (presumably because both tasks require a flexible switch between right and left body turns at the center of the maze), and its lesion caused a somewhat larger memory deficit when the animals had to switch quickly between tasks. These effects may be related to the position of the DSM as a functional intermediate between HPC and DSL circuits. Until recently, it was thought that the striatal-cortical loops operate in parallel, (Voorn et al., 2004), but a recent report (Aoki et al., 2019) described a functional multisynaptic and unidirectional connection from DSM-prelimbic loops to the striatal motor loops. HPC projects to the most medial part of DSM and to the medial prefrontal cortex (Groenewegen et al., 1987) and may then be able to influence activity in the DSL motor loops via DSM and the prefrontal circuits involved in decision-making. The DSL is not thought to be involved in flexible actions that depend on their outcome, although neurons in this area seem to respond to action value 
(Stalnaker et al., 2010), but, with extended training, overt motor actions come under DSL control through a process hypothesized to involve inhibitory control of the infralimbic over the prelimbic prefrontal cortex (Killcross and Coutureau, 2003). Although goal-oriented and habitual behaviors are generally considered to be distinct and to engage in competition, it has also been proposed that habits can be viewed as a sequence of actions under the control of a flexible reinforcement learning process (Dezfouli and Balleine, 2012). From this perspective, a stimulus may give rise to the urge to perform a certain action, but the execution does not proceed until evaluation takes place in the networks responsible for goal-directed behavior (Balleine, 2019). Thus, memory and decision processes are interconnected, but much remains to be elucidated about how stored information is eventually used to guide overt behavior. The current work strongly argues that understanding the functional significance of activity in a local memory circuit requires evaluation of that activity together with activity in other memory circuits and in light of the past experience of the organism.

In conclusion, the current data indicate that by using time and space, HPC and DSL can integrate cognitive and habitual memories in distinct and complementary fashion. This finding provides an articulated framework within which to further investigate the underlying brain mechanisms that lead to the formation of coherent, multifaceted memories, and underscores the degenerate nature of behavior. Together with previous research (Ferbinteanu, 2016), the current work helps to qualify the multiple memory systems paradigm. Different types of memories are indeed critically dependent on distinct neural circuits. In some situations, these circuits can operate independently and in parallel to guide behavior. However, in some other situations, the neural basis of a given type of memory can be greatly expanded across multiple memory structures and basic features of experience, such as time and space, are be used to integrate different types of memories within the same local network.

\section{References}

Aggleton JP, Pearce JM (2001) Neural systems underlying episodic memory: insights from animal research. Philos Trans R Soc Lond B Biol Sci 356:1467-1482.

Aoki S, Smith JB, Li H, Yan X, Igarashi M, Coulon P, Wickens JR, Ruigrok TJ, Jin X (2019) An open cortico-basal ganglia loop allows limbic control over motor output via the nigrothalamic pathway. eLife 8:e49995.

Balleine BW (2019) The meaning of behavior: discriminating reflex and volition in the brain. Neuron 104:47-62.

Cai DJ, Aharoni D, Shuman T, Shobe J, Biane J, Song W, Wei B, Veshkini M, La-Vu M, Lou J, Flores SE, Kim I, Sano Y, Zhou M, Baumgaertel K, Lavi A, Kamata M, Tuszynski M, Mayford M, Golshani P, Silva AJ, et al. (2016) A shared neural ensemble links distinct contextual memories encoded close in time. Nature 534:115-118.

Clark RE, Broadbent NJ, Squire LR (2005) Impaired remote spatial memory after hippocampal lesions despite extensive training beginning early in life. Hippocampus 15:340-346.

Devan BD, White NM (1999) Parallel information processing in the dorsal striatum: relation to hippocampal function. J Neurosci 19:2789-2798.

Dezfouli A, Balleine BW (2012) Habits, action sequences and reinforcement learning. Eur J Neurosci 35:1036-1051.

Eichenbaum H (2000) A cortical-hippocampal system for declarative memory. Nat Rev Neurosci 1:41-50.

Fellows LK (2018) The neuroscience of human decision-making through the lens of learning and memory. Curr Top Behav Neurosci 37:231-251.

Ferbinteanu J (2016) Contributions of hippocampus and striatum to memory-guided behavior depend on past experience. J Neurosci 36:64596470.

Ferbinteanu J (2019) Memory systems 2018: towards a new paradigm. Neurobiol Learn Mem 157:61-78
Gremel CM, Costa RM (2013) Orbitofrontal and striatal circuits dynamically encode the shift between goal-directed and habitual actions. Nat Commun 4:2264.

Groenewegen HJ, Vermeulen-Van der Zee ET, Te Kortschot A, Witter MP (1987) Organization of the projections from the subiculum to the ventral striatum in the rat. A study using anterograde transport of Phaseolus vulgaris leucoagglutinin. Neuroscience 23:103-120.

Groenewegen HJ, Voorn P, Scheel-Krüger J (2016) Limbic-basal ganglia circuits parallel and integrative aspects. In: The basal ganglia novel perspectives on motor and cognitive functions. Soghomonian J-J, ed), pp 11-45. Cham, Switzerland: Springer International Publishing.

Jacobson TK, Gruenbaum BF, Markus EJ (2012) Extensive training and hippocampus or striatum lesions: effect on place and response strategies. Physiol Behav 105:645-652.

Killcross S, Coutureau E (2003) Coordination of actions and habits in the medial prefrontal cortex of rats. Cereb Cortex 13:400-408.

Kim JJ, Baxter MG (2001) Multiple brain-memory systems: the whole does not equal the sum of its parts. Trends Neurosci 24:324-330.

Knowlton BJ, Mangels JA, Squire LR (1996) A neostriatal habit learning system in humans. Science 273:1399-1402.

Lee AS, Andre JM, Pittenger C (2014) Lesions of the dorsomedial striatum delay spatial learning and render cue-based navigation inflexible in a water maze task in mice. Front Behav Neurosci 8:42.

Lehmann H, Sparks FT, Spanswick SC, Hadikin C, McDonald RJ, Sutherland RJ (2009) Making context memories independent of the hippocampus. Learn Mem 16:417-420.

Mailly P, Aliane V, Groenewegen HJ, Haber SN, Deniau JM (2013) The rat prefrontostriatal system analyzed in 3D: evidence for multiple interacting functional units. J Neurosci 33:5718-5727.

Mankin E, Diehl G, Sparks F, Leutgeb S, Leutgeb J (2015) Hippocampal CA2 activity patterns change over time to a larger extent than between spatial contexts. Neuron 85:190-201.

McDonald RJ, White NM (1993) A triple dissociation of memory systems: hippocampus, amygdala, and dorsal striatum. Behav Neurosci 107:3-22.

McDonald RJ, White NM (1994) Parallel information processing in the water maze: evidence for independent memory systems involving dorsal striatum and hippocampus. Behav Neural Biol 61:260-270.

McDonald RJ, King AL, Hong NS (2001) Context-specific interference on reversal learning of a stimulus-response habit. Behav Brain Res 121:149165.

McGeorge AJ, Faull RLM (1989) The organization of the projection from the cerebral cortex to the striatum in the rat. Neurosci 29:503-537.

Murray EA, Wise SP, Graham KS (2016) The evolution of memory systems: ancestors, anatomy, and adaptations. Oxford, UK: Oxford UP.

O’Reilly KC, Alarcon JM, Ferbinteanu J (2014) Relative contributions of CA3 and medial entorhinal cortex to memory in rats. Front Behav Neurosci 8:292.

Packard MG, McGaugh JL (1996) Inactivation of hippocampus or caudate nucleus with lidocaine differentially affects expression of place and response learning. Neurobiol Learn Mem 65:65-72.

Packard MG, Hirsh R, White NM (1989) Differential effects of fornix and caudate nucleus lesions on two radial maze tasks: evidence for multiple memory systems. J Neurosci 9:1465-1472.

Paxinos G, Watson C (1988) The rat brain in stereotaxic coordinates, Ed 4. San Diego: Academic.

Ragozzino ME, Jih J, Tzavos A (2002a) Involvement of the dorsomedial striatum in behavioral flexibility: role of muscarinic cholinergic receptors. Brain Res 953:205-214.

Ragozzino ME, Ragozzino KE, Mizumori SJ, Kesner RP (2002b) Role of the dorsomedial striatum in behavioral flexibility for response and visual cue discrimination learning. Behav Neurosci 116:105-115.

Rubin A, Geva N, Sheintuch L, Ziv Y (2015) Hippocampal ensemble dynamics timestamp events in long-term memory. Elife 4:e12247.

Scoville WB, Milner B (1957) Loss of recent memory after bilateral hippocampal lesions. J Neurol Neurosurg Psychiatry 20:11-21.

Sharpe MJ, Stalnaker T, Schuck NW, Killcross S, Schoenbaum G, Niv Y (2019) An integrated model of action selection: distinct modes of cortical control of striatal decision making. Annu Rev Psychol 70:53-76.

Sparks FT, Lehmann H, Sutherland RJ (2011) Between-systems memory interference during retrieval. Eur J Neurosci 34:780-786.

Squire LR (2004) Memory systems of the brain: a brief history and current perspective. Neurobiol Learn Mem 82:171-177. 
Squire LR, Zola-Morgan S (1991) The medial temporal lobe memory system. Science 253:1380-1386.

Squire LR, Knowlton B, Musen G (1993) The structure and organization of memory. Annu Rev Psychol 44:453-495.

Stalnaker TA, Calhoon GG, Ogawa M, Roesch MR, Schoenbaum G (2010) Neural correlates of stimulus-response and response-outcome associations in dorsolateral versus dorsomedial striatum. Front Integr Neurosci 4:12.

Vicente AM, Galvão-Ferreira P, Tecuapetla F, Costa RM (2016) Direct and indirect dorsolateral striatum pathways reinforce different action strategies. Curr Biol 26:R267-R269.

Voorn P, Vanderschuren LJ, Groenewegen HJ, Robbins TW, Pennartz CM (2004) Putting a spin on the dorsal-ventral divide of the striatum. Trends Neurosci 27:468-474.

White NM, McDonald RJ (2002) Multiple parallel memory systems in the brain of the rat. Neurobiol Learn Mem 77:125-184
White NM, Salinas JA (2003) Mnemonic functions of dorsal striatum and hippocampus in aversive conditioning. Behav Brain Res 142:99-107.

White NM, Packard MG, McDonald RJ (2013) Dissociation of memory systems: the story unfolds. Behav Neurosci 127:813-834.

Yin $\mathrm{HH}$, Knowlton BJ (2004) Contributions of striatal subregions to place and response learning. Learn Mem 11:459-463.

Yin HH, Ostlund SB, Knowlton BJ, Balleine BW (2005) The role of the dorsomedial striatum in instrumental conditioning. Eur J Neurosci 22:513523.

Yin HH, Knowlton BJ, Balleine BW (2006) Inactivation of dorsolateral striatum enhances sensitivity to changes in the action-outcome contingency in instrumental conditioning. Behav Brain Res 166:189-196.

Yin HH, Mulcare SP, Hilário MRF, Clouse E, Holloway T, Davis MI, Hansson AC, Lovinger DM, Costa RM (2009) Dynamic reorganization of striatal circuits during the acquisition and consolidation of a skill. Nat Neurosci 12:333-341. 